Revista Iberoamericana. Vol. LXV, Núm. 186, Enero-Marzo 1999; 103-116

\title{
REGRESANDO A LA GUINEA: HISTORIA, RELIGIÓN Y MITO EN LAS NOVELAS CARIBEÑAS DE MAYRA MONTERO
}

\author{
POR \\ YAZmín PÉrez TORRES \\ Universidad de Puerto Rico, Arecibo
}

\begin{abstract}
The historical reality that all writers of the Caribbean must come to terms with is the cultural dislocation and fragmentation brought about by the European conquest of the Americas. Accordingly, the West Indian writer has all too often been paralyzed by a vision of the Caribbean as the site of historical and cultural shipwreck. Until very recently, writers of the West Indies overlooked the fact that the historic clash of cultures in the New World signaled not only the loss of a cultural heritage, but also the beginning of a new one.
\end{abstract}

Barbara J. Webb

I. Dislocación, REelaboración y Resistencia CUltural: LAS RELIGIONES AFRiCANAS EN LA AmÉrica Hispana

El fuerte enlace que desde sus mismos comienzos la escritura hispanoamericana establece entre historia y ficción ${ }^{1}$ trasbasa los límites temporales y discursivos de las así llamadas Crónicas de Indias para convertirse en uno de sus más importantes motivos recurrentes. Sobre todo desde el siglo XIX, cuando la inconformidad criolla con el régimen español desemboca en las independencias nacionales, muchos escritores han adoptado el papel de historiadores: han asumido la responsabilidad de escribir, de crear mediante su

\footnotetext{
' Después de haber superado las pretensiones de cientificismo y objetividad absoluta promovidas por las tendencias positivistas decimonónicas, al menos una parte de la crítica preocupada por la historiografía ha venido a aceptar que, al igual que la narrativa de ficción, la historia, la narrativa histórica, es esencialmente una construcción discursiva. En "The Discourse of History", Roland Barthes advierte que el discurso histórico es en esencia una forma de elaboración ideológica (16). Por su parte, en "The Historical Text" Hayden White sostiene que las narrativas históricas constituyen artefactos verbales, ficciones verbales cuyos contenidos son tanto inventados como encontrados y cuyas formas tienen más en común con la literatura que con las ciencias sociales (42). De hecho, lo que distingue a los relatos históricos de los ficcionales es su contenido más que su forma. Los primeros tienen como contenido sucesos reales, sucesos ocurridos; los segundos se nutren de sucesos imaginarios, inventados por el narrador (The content of the Form 27). La relación entre historia y ficción no es unívoca. Si la narrativa histórica se aproxima en sus métodos y procedimientos a la narrativa de ficción, resulta que la narrativa de ficción también se ha acercado conscientemente a aquélla en sus propósitos, pues ha pretendido "hacer" historia.
} 
escritura, la Historia, y por medio de este acto construir "la nación" propia ${ }^{2}$ o, en otras ocasiones, definir la especificidad del ser hispanamericano. Ahora como antes, este escritor/ historiador ha inventado su versión, ${ }^{3}$ aspirando a dar con ella una respuesta a la difícil pregunta de cómo escribir la historia americana: de cómo integrar sus dispares elementos en un relato que ofrezca una interpretación correcta y veraz de los acontecimientos que han marcado su devenir en el tiempo, y de su especificidad económica, social, política y cultural.

De los múltiples componentes de esa cambiante realidad americana, uno de los más difíciles de explicar era su variada composición racial y étnica. Efectivamente, "Una vez llegados los africanos al nuevo mundo, también ellos pasaron a formar parte del problema de cómo narrar la historia" (Garceau 76). Y a pesar de que la presencia del negro africano en América se remonta a principios del siglo XVI, Richard Jackson afirma que es a finales de ese siglo XIX, tan cargado de ideas del racismo científico, ${ }^{4}$ cuando se descubre esa presencia, y pasa a formar parte - junto con el indio y la tierra- de las preocupaciones del mundonovismo de principios de siglo XX (37). Esto se produjo, en parte, debido a una toma de conciencia de la realidad americana, y en parte por imitación de las tendencias artísticas e intelectuales europeas de postguerra, fascinadas con el mundo "primitivo y puro" que hallaban en Africa y en los pueblos del Pacífico.

La población negra africana, venida a América por medio de la trata esclavista, se concentró especialmente, aunque no exclusivamente, en el Caribe. Lo que para Sidney Mintz unifica este área geográfica, tan heterogénea en el aspecto cultural, son sobre todo los rasgos socio-estructurales que comparten sus países, el hecho de que basaban sus economías en el sistema de producción de la Plantación, lo cual la convierte en una societal area (1920). Por supuesto, quienes hacían funcionar la Plantación eran justamente los esclavos africanos, cuya presencia tuvo por consecuencia la "africanización" de la cultura caribeña (Benítez Rojo 43-9). Aunque en muchos casos no se puede hablar de conservación de formas africanas puras, sí se puede hablar de reelaboraciones ${ }^{5}$ de esas formas. Entre estos

\footnotetext{
${ }^{2}$ Benedict Anderson (Imagined Communities. Reflects on the Origin and Spread of Nationalisms), Homi K. Bhabha (Nation and Narration), Doris Sommer (Foundational Fictions. The National Romances of Latin America) y Jean Franco ("The Nation as Imagined Community"), entre otros, se han referido a la "nación" en términos de una comunidad imaginada, poniendo así de manifiesto lo que este concepto tiene de construcción discursiva e ideológica.

${ }^{3}$ Se ha dicho al respecto que "En el nuevo mundo el oficio de la historia jamás ha sido una actividad inocente" y que "La historia latinoamericana ha sido, desde el principio, una pugna entre diversas versiones de la historia" (Garceau 75-6).

4 "Negative opinions about dark-skinned peoples in the late nineteenth century were strongly influenced by European racist theories such as those in Count Gobineau's classic Essay on the Inequality of Human Races that coincided with the racist opinions of New World intellectuals who, inheriting the old prejudices of the colonial ruling class, were convinced of the inferiority of the darker races. With amazing ease the Europeans versions of racism were applied by homegrown racists to the multiracial environment of the Americas" (Jackson 36).

${ }^{5}$ De acuerdo con Knight and Crahan "[...]despite what Melville Herskovits and others have implied, cultural legacies from Africa cannot be traced in a linear fashion foward or backward. Mintz and Price deal with this problem in great detail, concluding that 'if Afro-American cultures do in fact share such an integral dynamism, and if [...] their social systems have been highly responsive to changing social
} 
elementos culturales reelaborados en América, uno de los más importantes y poderosos, si no el más, lo constituyen las religiones africanas, principalmente porque

Las variables de la religión negroamericana, con mayor o menor reelaboración de los modelos africanos, se convirtieron en una supereestructura religiosa que dio significado y permitió la sobrevivencia física y espiritual de importantes sectores de la población negra en las Américas y que sirvió de respuesta antitética al paternalismo que la Iglesia cristiana ayudaba a imponer como institución del sistema etnocéntrico oficial (Dos Santos y Dos Santos 104).

El refugio que las religiones africanas ofrecieron a los negros en América no se limitó a la época colonial, sino que su fuente de resistencia física y cultural se extiende hasta el presente. El mejor ejemplo de este fenómeno es el caso de Haití, en donde el vudú, religión más popular en el país, ha servido para unificar a los esclavos que pelearon y ganaron la revolución haitiana, y para cohesionar la oposición al depuesto régimen del dictador JeanClaude Duvalier. ${ }^{6}$ Pero su importancia no se limita a este aspecto porque

La religión en el Africa negra no es cosa que pueda separarse del conocimiento, de la política, de la economía, de lo social o de lo cultural; no es posible siquiera distinguirla de la historia, puesto que ella misma es historia: se trata de un discurso que permea [sic] toda la actividad humana e interfiere en todas las prácticas (Benítez Rojo 162).

El igualamiento de la religión africana y la historia ofrece un punto de partida propicio para realizar un acercamiento a una parte de la obra novelesca de la escritora Mayra Montero, quien en dos de sus novelas La trenza de la hermosa luna (1987) y Del rojo de su sombra (1992), ha explorado, entre otros asuntos, la relación entre el vudú y la historia en el Caribe. ${ }^{7}$

II. Historia, RELIGIÓN Y MITO EN LA TRENZA DE LA HERMOSA LUNA Y DEL ROJO DE SU SOMBRA, DOS novelas "haitianas" De Mayra Montero

En un sentido amplio del término, ${ }^{\circledR}$ estos dos textos pueden ser considerados novelas históricas. A pesar de no poder hallarse en ellos abrumadoras referencias a fechas, personajes o datos históricos específicos, sí están basados en situaciones y acontecimientos

conditions, one must mantain a skeptical attitude toward claims that many contemporary social or cultural forms represent direct continuities from the African homelands"' (9).

${ }^{6}$ Para un estudio completo acerca del origen del vudú en Haití y su papel dentro de la política y la sociedad haitiana, véase el libro de Michel Laguerre Voodoo and Politics in Haiti.

${ }^{7}$ Mayra Montero es, además, autora de las novelas La última noche que pasé contigo y Tú, la oscuridad.

${ }^{8}$ Se emplea aquí la definición que Danielle Aubin toma prestada a Leon Hoffman para acercarse a la novela histórica antillana: "un roman dans lequel des événements précis tirés de l'Histoire déterminent ou influencent le déroulement de la trame et lui servent dans une large mesure de cadre référentiel" (31). 
ocurridos en la vida de Haití y República Dominicana. Y en última instancia, y más importante aún, estas obras ofrecen aquello que brinda "la novela histórica" en general: una visión de las mentalidades, valores y estructuras de pensamiento ("Fuego cruzado" 85) de una época; y además

[...] une sorte de laboratoire dont les expériences peuvent provoquer l'emergence, au sein de l'historiographie établie à prétention objective, de nouveaux modèles pour penser l'histoire [...] (Lüsebrink 127).

La trenza de la hermosa luna recoge el clima de tensión social y política que se vivió en Haití en las vísperas de la caída de Duvalier hijo. El relato, narrado por una voz omnisciente, está focalizado a través del protagonista Jean Leroy, marinero de goleta que regresa a Haití, su país natal, tras haberse ausentado por veinte años, al recibir un llamado de su mejor amigo de la infancia, Papá Marcel, ahora convertido en un houngán del vudú. Lo que se narra es justamente ese retorno que lo trastorna al enfrentarlo con sus recuerdos y con una realidad que casi siente ajena por lo confusa y caótica que se le muestra.

Con el regreso a su tierra, se inicia en la vida de Jean Leroy un difícil proceso de cambios, conducentes a un reconocimiento y una toma de posición, que provee la materia prima para el desarrollo del drama psicológico alrededor del cual se construye el texto.

El primer escalón en este proceso, que en alguna medida constituye un movimiento en ascenso, es la sorpresa, el asombro, la incapacidad de reconocer lo que encuentra a su llegada." Luego se da un intento fallido por reanudar parte de la vida que abandonó y que ya ha sido tragada por un pasado perdido e irrecuperable. ${ }^{10}$ El sentimiento de extrañeza que le produce la realidad caótica de su país, a la que había estado ajeno por tanto tiempo, y el sentimiento de derrota al no poder recuperar su pasado, provocan en Jean Leroy el deseo de huir y olvidar para siempre esa tierra en la cual ya no halla cabida. Pero la brutalidad de la situación haitiana, paladeada apenas por unos pocos días, y el haberse involucrado de alguna manera en la resistencia popular contra el régimen son factores que contribuyen a la formación de una conciencia que aflora en un momento crucial: cuando decide quedarse en Haití y luchar por la reconstrucción nacional.

Este proceso de toma de conciencia y de compromiso por el que atraviesa Jean Leroy provee obviamente un medio para la denuncia política y, en un menor grado, la denuncia social. La opresión política, puesta de manifiesto en las muertes y los incontables atropellos de los tonton macoutes, la miseria económica generalizada y el sentimiento popular de no estar representados por un gobierno "de mulatos" se han conjugado para crear una situación en la cual el régimen de Duvalier no se sostiene más. Como consecuencia directa de esto surgen en el país varios tipos de oposición organizada y es en este contexto que la religión

\footnotetext{
${ }^{9}$ Puesta de manifiesto desde el mismo comienzo, con su dificultad de identificar los rostros demudados de sus propios amigos en la reunión convocada por Papá Marcel.

${ }^{10}$ Jean Leroy trata de reanudar su antigua relación amorosa con Choucoune, interrumpida por su partida; y por otra parte, desearía que su relación con Papá Marcel fuera igual que cuando eran niños, cuando su admiración por el houngán no estaba sombreada por la duda de su complicidad con el sistema político opresor.
} 
vudú cobra un lugar prominente en el texto, que difiere ostensiblemente del papel de mero telón de fondo para la acción novelesca que se le ha atribuido."

El primer tipo de oposición es la que practican los jóvenes, quienes no parecen tener una fuerte vinculación religiosa y sus métodos de acción son más abiertos, seculares y tradicionales. Participan en manifestaciones de protesta, en las "candelas" o quemas callejeras y se enfrentan, con tiros y piedras, al ejército. En cambio, el segundo tipo de oposición hallada en el relato es la de Papá Marcel y la red de hounganes y colaboradores, que trabajan con los medios que les ofrece su fe religiosa. La Operación Bambú, de la cual participaba Jean Leroy, consistía en la distribución secreta, por todo el país, de unos polvos venenosos preparados según la farmacopea vuduista, con los cuales se intentaba matar a los asesinos tonton macoutes.

Pero lo cierto es que en el texto la religión no se representa de forma unidimensional. Aquí la novela se atiene a lo que estudiosos de diversas disciplinas académicas han señalado con relación al ambiguo papel del vudú en la historia y la política de esa nación en el siglo actual. El doble juego de apoyo y resistencia a los distintos régimenes de gobierno que ha jugado a través de la historia (Laguerre 100-20), lo encarnan en el texto los personajes del houngán Toussaint Rigaud, colaborador incondicional de "Papa Doc", y su hijo el houngán Marcel Rigaud, cuya bien lograda caracterización no permite que su verdadera actitud política se llegue a conocer con plena certeza. ${ }^{12}$

La aceptación de esta dualidad del vudú en la historia política de Haití cumple con una función vital en el texto porque aparte de que ayuda a forjar la base histórica (necesaria para que éste constituya una novela histórica) evita que ésta caiga en la trampa de una peligrosa mitificación de la religión afrocaribeña, aún cuando resulta evidente que, al incluirla dentro de los agentes modeladores del devenir histórico de Haití, se intenta legitimar su existencia y afirmar su validez ante una perspectiva occidental dominante que ha intentado negarla desde su mismo origen. También cabe señalar que el relato se rehusa a utilizar la religión vudú para hacer de Haití meramente un mundo exótico - lo cual sería una mera proyección de prejuicios occidentales con respecto a gentes de origen no occidental- pues todos esos ingredientes que podrían pasar ante el lector occidental por elementos "sobrenaturales" (las capacidades adivinatorias y curativas del houngán Papá Marcel, por ejemplo) son, al fin de cuentas, parte del sistema de creencias que constituyen el vudú y las religiones afroamericanas en general. ${ }^{13}$

Dada la evidente preocupación por la situación histórica y política concreta por la que atraviesa Haití en un momento específico, La trenza de la hermosa luna se inscribe dentro de la tendencia de las novelistas contemporáneas, señalada por Linda Anderson, que no escriben sobre ¿qué es la historia?, sino que tratan de reclamar el pasado en nombre de los silenciados y los marginados por la misma (132).

\footnotetext{
"Cuando hablo de la consideración del mundo religioso haitiano como telón de fondo, me refiero, paradójicamente, a las acotaciones de la contraportada que acompañan al texto en su primera edición. ${ }^{12}$ ¿Acaso se puede afirmar que es cómplice de las atrocidades de su padre; son auténticos su deseo de cambio y su participación activa en el derrocamiento de "Baby Doc"?

${ }^{13}$ Sería interesante estudiar qué relación existe, si alguna, entre la tradición del realismo mágico, según la entienden Miguel Ángel Asturias y Alejo Carpentier, y los textos novelescos de Mayra Montero que se ocupan del tema del vudú.
} 
Tomando en cuenta la manera en que se presentan los hechos en el texto, se podría afirmar que la concepción de la historia subyacente al mismo es la que, con su origen, define y marca el inicio de la edad moderna: la historia como un devenir de hechos transcurridos en un tiempo lineal, irrepetible y progresivo (Benedict Anderson). Los acontecimientos que se narran en el texto vienen como consecuencia lógica del entrejuego de fuerzas económicas, políticas, sociales y culturales. Con la caída de Duvalier se marca el final de toda una era, de un período histórico, y se vislumbra el comienzo de otro que quizás podría ser mejor: uno en el cual el hombre y la mujer haitianos tienen la oportunidad, aunque sea remota, de construir su futuro. Por eso se queda Jean Leroy en Haití, para tomar las riendas de ese proceso difícil y necesario. Por eso al final se queda el lector con un dejo de esperanza.

La novela Del rojo de su sombra muestra marcadas diferencias. Lo primero que le llama la atención al lector al enfrentarse con el texto es una "Nota de la autora" que pretende anteceder al relato, pero que forma parte de éste de todas maneras, y convierte a "la autora" en otra voz narrativa más dentro de la ficción. Este apartado resulta importante pues en él se aclara que la novela está basada en hechos ocurridos "en la vida real", con lo cual se establece desde el comienzo el carácter verídico, "histórico", del relato. También resulta curiosa la forma en que mezcla la denuncia social, el discurso etnográfico y el discurso policial para constituir una especie de guía al lector, que convierte al relato en una especie de crónica de una muerte anunciada. Lo más revelador de todo, sin embargo, son las últimas palabras de esta nota aclaratoria, pues dan la clave para la línea de interpretación que se sigue aquí:

Esta novela narra los hechos verídicos ocurridos hace pocos años en algún punto de La Romana. [...] Los nombres y algunos lugares han sido alterados en beneficio de los informantes. Detrás de un caso que la policía dominicana cerró como un simple "crimen pasional", palpita el hechizo de una guerra que aún no termina de pelearse (10, énfasis mío).

De acuerdo con esta confesión, detrás de los puros hechos históricos hay algo más. Los acontecimientos que se narran aquí - la vida y la muerte de Zulé Revé, dueña de uno de los Gagás $^{14}$ organizados y mantenidos por los braceros haitianos en las plantaciones de caña de la República Dominicana, al enfrentarse con otro dueño de Gagá, el temible Similá Bolosse-, esa guerra aún inacabada, van revestidos de una trascendencia que rebasa lo meramente histórico para adentrarse en el plano de lo mítico.

El primer elemento que se podría utilizar para relacionar esta novela con el mito son las características que Mircea Eliade atribuye a la "conducta mítica", típica de los "pueblos primitivos": "extrañas formas de comportamiento" y "excesos" (El mito del eterno retorno 3). Eso es justamente lo que esta narración entrega al lector: la extrañeza y lo excesivo de todo ese mundo en el que los personajes viven submersos.

\footnotetext{
${ }^{14}$ En el artículo periodístico "El Gagá de Similá” Mayra Montero define al gagá como un culto socioreligioso practicado por haitianos y dominicanos en las zonas cañeras de la República Dominicana (10). Los ritos del gagá, relacionados a las prácticas del vudú, fueron traídos a la región por los trabajadores de la caña haitianos. Con el tiempo han incorporado elementos de otras religiones y del folclor dominicano (10).
} 
Excesiva es antes que nada la miseria que los arropa. El escenario y el ambiente donde se desarrolla la acción dan pie a una fuerte denuncia de la condición social y económica infrahumana en la que (sobre)vive esta población.

Aguantarían allí lo que tuvieran que aguantar hasta que la sequía se aliviara y el cañaveral volviera a sus cabales. Papá Luc y los muchachos mayores se irían a chapear a La Romana o a recoger la piña en los alrededores de Bonao, y así levantarían unos centavos para mandarle a Anacaona. Ella y el hijo más pequeño vivirían mientras tanto de la poca yuca que les quedaba enterrada, de unos puñados de harina atizonada y floja, y sobre todo del aire (60).

De hecho, en el texto se hallan varias referencias que igualan esta situación a la esclavitud que vivieron sus antepasados de origen africano.

La dueña [...] le había oído decir a su tío que el mandamás de Port-au-Prince le vendía braceros al mandamás de la Dominicana. Los vendía a ochentaicinco pesos la cabeza, a veces por menos. Muchos braceros, miles de miles, y, cuando ya no había cabida para ellos en los ingenios del Gobierno, venían los traficantes de los demás centrales y los compraban más baratos, un puñado de negros por aquí, otro puñado por allá, aquellos congos medio muertos que iban de un lado para otro como ganado medio vivo (125).

Y otra vez, como en tiempos de la esclavitud original, en medio de esta precariedad los braceros inmigrantes recurren, entre otras cosas, a sus creencias religiosas como medio de supervivencia espiritual y cultural. Por eso, las vidas de los personajes giran alrededor de la religión vudú.

Excesivo es también por demás el comportamiento de los personajes, sobre todo el de los protagonistas. Pero estos se explican si se consideran a la luz de la definición del mito ofrecida por Eliade:

In general it can be said that myth, as experienced by archaic societies, [1] constitutes the History of the acts of the Supernaturals; [2] that this History is considered to be absolutely true (because it is concerned with realities) and sacred (because it is the work of the Supernaturals); [3] that myth is always related to a "creation," it tells how something came into existence, or how a pattern of behavior, an institution, a manner of working were established; this is why myths constitute the paradigms for all significant human acts; [4] that by knowing the myth one knows the origin of things and hence can control and manipulate them at will; this is not an "external," "abstract" knowledge but a knowledge that one "experiences" ritually, either by ceremonially recounting the myth or by performing the ritual for which it is the justification; [5] that in one way or another one "lives" the myth, in the sense that one is seized by the sacred, exalting power of the events recollected or re-enacted (Myth and Reality 19). ${ }^{\text {is }}$

15 Barbara J. Webb destaca la complejidad del concepto de mito y apunta al hecho de que sus usos, por varias disciplinas, es a veces contradictorio. Así, compara las definiciones ofrecidas por varios estudiosos del mito: 
Desde el comienzo del relato se sugiere, de alguna u otra forma, que los acontecimientos a ser narrados ocurren inevitablemente, como cumplimiento de un destino predeterminado por fuerzas superiores al designio y la voluntad humana. La brava y terca Zulé, en cuya vida se cumple ese destino, insiste en ir a su encuentro con Similá a pesar de que sabe, aunque sea por intuición, que en él hallará la muerte. Son muchas las fuentes que le transmiten ese mensaje al que ella hace caso omiso. Lo anuncia la naturaleza (el calor inusual; el olor a sal mojada y a pescado que se sentían el día de la partida del Gagá) y se lo advierten sus seres queridos (Papá Luc, Honoré Babiole y Jérémie Candé le advierten que Similá ha jurado matarla; que se ha bañado en la sangre de cien cabritos para que el loa Lokó Siñaña lo ayude en sus propósitos; que viene armado, y que viene acompañado por una turba de tonton macoutes tan feroces como él). Sin embargo, como era de esperarse, la dueña resuelve seguir adelante con su peregrinación y decide que si tiene que morir, morirá. De este momento en adelante todos comparten un sentimiento de lo inevitable, de fatalidad, expresado por Jérémie Candé

-Eres terca, dueña Zulé. Pero eso no es nada nuevo. Lo que sí me asombra es que estés arrastrando a tanta gente a una bronca que ya está perdida (83).

y, más claramente aún, por la voz narrativa que interpreta los sentimientos del padre de Zulé

Papá Luc, que los observa desde lejos, viene dispuesto a intervenir, pero le basta una fina mirada de Zulé para comprender que todo está hecho, sellado y sumergido por el tirón eterno de un pedral de ley (103).

Pero lo que realmente convierte en inevitable ese encuentro con la muerte es el carácter profético que reviste, adquirido cuando al momento de su muerte, Papá Coridón le advierte a Zulé que si no se casa con Jérémie, debe cuidarse del próximo hombre que vendrá a su vida, que no es otro que Similá Bolosse.

Por otra parte, a través de todo el relato resulta evidente el gran peso que tienen los elementos "sobrenaturales" tanto en la construcción de personajes como en la acción novelesca. Vital importancia, en ese sentido, tiene la lluvia de peces:

En época de la Cuaresma y hasta el final de la Semana Santa, los aguaceros que caen por los alrededores de la Colonia Engracia huelen perdidamente a mar. A veces les han venido peces con la lluvia, una especie de sardinas que se quedan coleteando un rato sobre el fango y al día siguiente son ya carroña fácil, inundando con su muermo espantoso todos los

\footnotetext{
"The original definitions of myth is narration or story. Myth are usually considered the spontaneous expression of a people, associated with religious beliefs and ritual; thus, Mircea Eliade's definition of myth as 'sacred stories'. Isidore Okpe who, however, rejects the dichotomy between sacred and secular used to distinguish myth from other popular traditions, such as fables, folktales, and legends; he emphasizes the oral and inventive aspects of these communal narratives. Similarly, G. S. Kirk challenges the notions of myth that have developed over the last century and emphasizes the speculative, imaginative content of myth. This type of orally transmitted narrative speculates on the unknown or some as-yet unresolved problem of a community" (5).
} 
rincones del batey. Los braceros más viejos todavia se acuerdan de aquel día en que un tornado mejor bragado que los otros soltó en el campo un tiburón pequeño y ya cadáver, un animal de acero que aún apretaba entre los dientes el pescuezo imposible de un pajarito (55).

Nuevamente, más que pintoresquismo tropical o exotismo decorativo, lo "sobrenatural" ligado a lo mítico-religioso tiene una función eminentemente pivotal: dotar al relato de un carácter profético, cosmogónico-apocalíptico, sobre el devenir histórico de un pueblo. Esa imagen de los seres acuáticos caídos del cielo reaparece en la canción que canta el Gagá al alistarse para partir en peregrinaje por las distintas plantaciones.

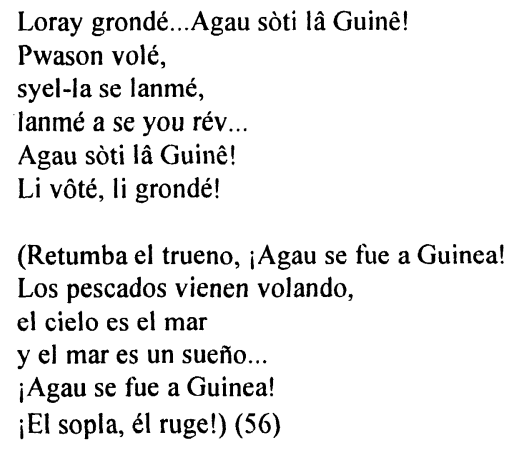

Aquí la narración se focaliza a través de un personaje colectivo, los miembros del Gagá, que no hacen una clara distinción entre una realidad objetiva, empírica, y el contenido de sus mitos, porque su concepción de la realidad es mítica. Esto se puede inferir del hecho de que sean los braceros más viejos los que "recuerdan" el tiburón venido con la lluvia y transmiten ese recuerdo a los más jóvenes, con lo cual se crea la tradición oral y el mito. De ahí que la canción, esencial dentro del ritual religioso y dentro de las prácticas de conservación y reelaboración cultural, hable de un retorno (el eterno retorno) (Eliade, El mito) a la Guinea - lugar de utópica libertad dentro del imaginario de los esclavos africanos en el nuevo mundo- y de un sueño.

En cuanto a lo "sobrenatural" en los personajes, Zulé, al igual que todos los hounganes y dueñas de gagás, posee facultades adivinatorias, curativas y tiene la capacidad de influir en la naturaleza. Similá tiene, además la capacidad de estar en todas partes y posee una característica única: tiene tres testículos. Aparte de eso, el carácter y la conducta de ambos obedece al carácter y a la conducta típica de las loas o divinidades vuduistas que ellos encarnan al ser montados.

Antes de que aparezca el personaje de Similá en la acción, ya el lector lo conoce por referencias de otros personajes

—Pues entonces cuídate del que venga luego - musitó [Coridón] con la garganta demolida. Tiene tres bolas, como la bestia negra de Jacmel. Cuidate de su meaja (78). 
o de la voz narrativa

Luego el bokor de Paredón se fue por donde habia venido y no volvió a saberse de él hasta que ya era dueño de Gagá de la Colonia Tumba; servidor de casta para Olisá Bayí; potro de guerra para Caé Samá; y voz y cuerpo y pura sangre para su jinete más frecuente y obstinado, que no podía ser otro que aquel llamado Toro Belecou (63).

Más adelante se deja saber que esa loa era "el misterio más temido y rencoroso del Panteón" vuduísta. El mismo Similá asume esta identidad al cantar

\author{
M'tòro m'béglé, \\ nâ savân mwê... \\ Tòro mwê tòro, sa ki mâdé pu mwê, \\ ou a di o \\ mwê mèm kriminel.
}

(Soy un toro,

bramo en mi sabana...

Toro, soy un toro,

al que pregunte por mí

dile que soy un criminal) (96).

En el caso de Zulé, además de caracterizarse por su fiereza, su valentía, desde el comienzo de la narración se insiste en su sexualidad desenfrenada, lo cual más adelante la relaciona con la loa que ella encarna

Luego lo vio partir, cubierta ella también por la lana de los vientos, pero sin voz suficiente para reclamarlo, llorosa y puta como la metresa Freda, sumisa y grande como la Virgen de Erzulie (96).

Pero estas equiparaciones no cobran entera significación sino hasta el final del relato, cuando por fin se produce el anunciado encuentro. En este momento los protagonistas asumen enteramente las identidades de los susodichos misterios

Similá Belecou, gran animal del campo, mejor conocido por Toro Bolosse, fiero señor del mundo, levanta su machete y escupe tres palabras sucias de las que les encantan a los dioses bravos (168-9).

La hija de Papá Luc, con la navaja en alto y la pelambrera suelta se parece más que nunca a la metresa Erzulie Freda, puta caliente del corazón profundo, amante de perfumes y de condumios blancos, de todo lo que tenga harina y todo lo que huela a leche. [...] Zulé echa a caminar hacia el bokor, sumisa como si le hubieran ofrecido un refresco amarillo, un cigarrillo mentolado, una enorme verga retinta, todo lo que más le gusta a la metresa Freda (171).

También en ese momento el lector se entera de la antigua contienda de ambas divinidades, al ser contada su historia por la voz narrativa. 
Cuentan los santos que la metresa Freda se empeñó en probar la meaja de Toro Belecou. Pero Toro Belecou se dedicó a humillarla, la maltrató en las noches y la obligó a beber de los orines blancos que desaguaban por aquellos tiempos las culebras negras.[...]

Erzulie Freda decidió vengarse. Caminó entonces en la noche y buscó en el monte al más zafio de todos los loases, uno que llaman Belie Belcan; le ofreció un chivo, que es lo que más le gusta, y le rogó que le hiciera un daño de revolcadero a Toro Belecou. Belie Belcan aceptó la ofrenda, aseguró que a cambio le traería la sangre de aquel toro. Pero lo cierto es que jamás cumplió (171).

Es indudable que ese "Cuentan los santos" con que comienza esta relación localiza la acción narrada en los inciertos tiempos primigenios y convierte al relato en una "historia sagrada". Con esto consigue otorgarle el carácter mítico que se le puede atribuir. Visto de este modo, el empeño de Zulé por enfrentarse con Similá se puede considerar como un intento de completar la venganza de Erzulie Freda, de hacer justicia ante los abusos del Toro Belecou. Pero como estaba escrito - inscrito, por el mito, en el inconciente colectivo de ese puebloZulé no podía salirse con la suya. Por eso muere a manos de Jérémie Candé, quien también encarna a otra deidad vuduista, y que se podría ver como el que desempeña el papel de Belie Belcan, el misterio que no le cumplió su promesa a la loa enamorada. En otras palabras, la relación amorosa entre Zulé y Similá y su trágico final vienen a ser una de las necesarias repeticiones o reactuaciones, (re-enactments) de un mito - exigidas por la negación de la irreversibilidad de los acontecimientos, correspondiente a la concepción temporal típica de las sociedades "arcaicas", según Eliade - que al fin de cuentas trata sobre la lucha entre dos opuesto universales: las fuerzas del mal y las fuerzas del bien.

Como último ejemplo del carácter mítico de los hechos que se narran en la novela, cabe argüir la circularidad de la vida de Zulé en la República Dominicana: comienza recibiendo un baño de su madrastra Anacaona y termina de la misma manera. Esto remite al eterno retorno, a la continua repetición de los mismos acontecimientos, típica de una concepción mítica de la realidad. El hecho de que Similá haya salido ileso e impune del enfrentamiento es lo que ofrece la posibilidad de que se repita la misma situación de explotación en el futuro. Lo cual seguramente ocurrirá, porque si bien es cierto que todo esto sucede por designio divino, también es cierto que en la fïgura de Similá Bolosse está representada toda la opresión a la que se han visto sometidos los haitianos y que los persigue como una maldición a donde quiera que vayan. Eso explica tal vez el tono pesimista y el sabor amargo que deja en el lector.

\section{A MANERA DE CONCLUSIÓN}

Existe una estrecha relación entre el mito y la historia. De acuerdo con William H. Mc Neill (en Lincoln), por ejemplo, ambos tratan de explicar el porqué del estado de cosas mediante el relato de algún tipo de historia ("story"). Comunmente, se tiene al primero por falso y a la segunda por cierta. (1) Bruce Lincoln, por su parte, distingue esas dos categorías no por su contenido; sino por los alegatos de veracidad que hacen sus narradores y por el tipo de recibimiento que la audiencia les brinda. En ese sentido, una narración que alegue veracidad, pero que carezca de credibilidad por parte del receptor sería una leyenda, 
mientras que la que sí goza de credibilidad sería Historia. El mito se diferencia de la Historia porque, además de reclamar veracidad y de disfrutar de credibilidad, posee autoridad. Es decir, que su verdad pretende ser paradigmática (24). La frecuente repetición de un mito puede ayudar a mantener el status quo de una sociedad ${ }^{16} \mathrm{o}$, por el contrario, puede colaborar con la construcción de nuevas formas sociales (25). Dada esa poderosa facultad no es difícil percibir su importancia.

Las novelas La trenza de la hermosa luna y Del rojo de su sombra, están estrechamente unidas por el acercamiento a distintos elementos de la realidad histórica contemporánea del pueblo haitiano, partiendo desde perspectivas diferentes. Por eso, si bien la primera se ocupa más que nada de una representación "objetiva" de la situación política en Haití en un momento histórico específico; y la última se interesa por lo mítico — sin dejar de lado el plano de la opresión social- visto a través de los haitianos emigrantes a la República Dominicana, se puede afirmar que ambas se complementan mutuamente, constituyendo así las dos caras de la misma moneda: la historia de explotación, sufrimiento y lucha del pueblo haitiano.

Quizás no se pueda hablar, con relación a estas novelas, de un intento deliberado de redefinir la "historia" o de reescribir la totalidad de "la historia haitiana", dado lo específico de los temas y las situaciones que aborda. Sin embargo, vistas en conjunto, y considerando sobre todo el lugar preeminente que ocupa en los textos la religión vuduista -elemento que jalona el devenir histórico de Haití- sí constituyen, inevitablemente, una reescritura o una (re)construcción de esa Historia.

La respuesta que la escritura de Mayra Montero da a la pregunta de cómo escribir la historia haitiana - y por extensión la caribeña - es alternativa y revolucionaria en cuanto se centra en los seres que han sufrido la dislocación y la fragmentación resultantes de las empresas imperialistas de Occidente; y al hacerlo no los acultura ni les niega un poder de creación, sino que parte desde las reelaboraciones culturales y mecanismos de resistencia que les han permitido la supervivencia.

Lo que habría que meditar es cómo interpretar la oposición que existe entre los finales de ambas novelas. A este respecto habría que preguntarse si se pueden ver como complementarios - con lo cual se daría la idea de que la maldición de la historia haitiana revelada por Del rojo de su sombra puede ser revocada, que el destino del haitiano está por construirse todavía, y que él tiene el poder para hacerlo, como propone La trenza de la hermosa luna - o si por el contrario laúltima novela constituye un mentís y una cancelación de la esperanza que se trasluce en la primera; si es la historia haitiana y caribeña en proceso de deconstrucción.

\section{OBRAS CITADAS}

Anderson, Benedict. Imagined Communities. Reflects on the Origin and Spread of nationalism. London: Verso, 1991.

${ }^{16}$ En relación con esta función, Roland Barthes ha afirmado que el mito tiene la tarea de otorgarle una justificación natural a una intención histórica y lograr que lo que es contingente parezca eterno (Lincoln 5). 
Anderson, Linda. "The Re-Imagining of History in Contemporary Women's Fiction". Plotting Change: Contemporary Women's Fiction. London: Arnold, 1990.

Aubin, Danielle. "Approche du roman historique antillais". Presence Africaine: Revue Culturelle du Monde Noir/Cultural Review of the Negro World 148 (1988): 30-43.

Barthes, Roland. "The Discourse of History". Comparative Criticism: A Yearbook 3 (1981): 3-20.

Bhabha, Homi. Nation and Narration. London: Routledge, 1990.

Benítez Rojo, Antonio. La isla que se repite. El Caribe y la perspectiva posmoderna. Hanover: Del Norte, 1989.

Dos Santos, Juana Elbein y Deoscoredes M. Dos Santos. "Religión y cultura negra". Africa en America Latina. Manuel Moreno Fraginals, ed. New York: Holmes \& Meier, 1984.

Eliade, Mircea. El mito del eterno retorno. Arquetipos y repetición. Madrid: Alianza, 1984.

Myth and Reality. New York: Little, 1968.

"Fuego cruzado. La novela y la historia en el Caribe." Actividad que se llevó a cabo en la Universidad Metropolitana el jueves 15 de marzo de 1990.

Garceau, Linda. "Socrates Yerbero: los negros y la historia en El siglo de las Luces de Alejo Carpentier". Filología 22 (1987): 75-99.

Jackson, Richard L. The Black Image in Latin American Literature. Albuquerque: University of New Mexico Press, 1976.

Knight, Franklin W. y Margaret E. Crahan (eds.). "The African Migration and the Origins of an African-American Society and Culture". Africa and the Caribbean. The Legacies of a Link. Baltimore: Johns Hopkins University Press 1979.

Laguerre, Michel S. Voodoo and Politics in Haiti. New York: St. Martin's Press, 1989.

Lüsebrink, Hans-Júrgen. "De l'incontournabilité de la fiction dans la connaissance historique. Questionnements théoriques à partir de romans historiques contemporains d'Alejo Carpentier, de Yambo Ouologuem et d'Ousmane Sembène". Neohelicon 16 (1986): 108-28.

Mintz, Sidney W. "The Caribbean as a Socio-cultural area". Peoples and Cultures of the Caribbean. Michael M. Horowitz, ed. New York: The Natural History Press, 1971. 17-46.

Montero, Mayra. Del rojo de su sombra. Barcelona: Tusquets, 1992. "El gagá de Similá". El Mundo (Puerto Rico Ilustrado). 21 de mayo de 1989. 10. La trenza de la hermosa luna. Barcelona: Anagrama, 1987. La última noche que pasé contigo. Barcelona: Tusquets, 1991. Tú, la oscuridad. Barcelona: Tusquets, 1995.

Sommer, Doris. Foundational Fictions. The National Romances of Latin America. Berkeley: University of California Press, 1991.

Webb, Barbara J. Myth and History in Caribbean Fiction: Alejo Carpentier, Wilson Harris, and Edouard Glissant. Amherst: University of Massachusetts Press, 1992.

White, Hayden. The Content of the Form. Narrative Discourse and Historical Representation. Baltimore: Johns Hopkins University Press, 1987.

"The Historical Text as Literary Artefact". The Writing of History. Literary Form and Historical Understanding. Robert H. Canary y Henry Kozicki, eds. Madison: University of Wisconsin Press, 1978. 41-62. 
Zimra, Clarisse. "W/Righting His/tory: Versions of Things Past in Contemporary Caribbean Women Writers". Explorations: Essays in Comparative Literature. Makoto Ueda, ed. Lanham, MD: University Press of America, 1986. 227-52. 